\title{
Walsh Transforms, Balanced Sum Theorems and Partition Coefficients over Multary Alphabets
}

\author{
M. T. Iglesias \\ Dept. of Mathematics \\ Universidade da Coruña \\ A Coruña, Spain \\ totero@udc.es \\ A. Verschoren \\ Dept. of Math. and C. Science \\ Universiteit Antwerpen \\ Antwerp, Belgium \\ alain.verschoren@ua.ac.be
}

\author{
B. Naudts \\ Dept. of Math. and C. Science \\ Universiteit Antwerpen \\ Antwerp, Belgium \\ bart@naudtsconsulting.com \\ C. Vidal \\ Dept. of Computer Science \\ Universidade da Coruña \\ A Coruña, Spain \\ conchi@dc.fi.udc.es
}

\begin{abstract}
In this note, we indicate how the basic machinery of Walsh transforms can be generalized from the binary case $([3,4])$ to multary alphabets. Our main results show how Walsh coefficients are related to partition coefficients and how they may be used to calculate schema averages.
\end{abstract}

\section{Categories and Subject Descriptors}

I.2.8 [Artificial Intelligence]: Problem Solving, Control Methods, and Search; F.2.2 [Analysis of Algorithms and Problem Complexity]: Nonnumerical Algorithms and Problems.

\section{General Terms}

Algorithms, Theory.

\section{Keywords}

Genetic algorithm, schema theory, Walsh function, Walsh coefficient, partition coefficient, schema average, multary alphabet.

\section{INTRODUCTION}

It is well-known that the ordinary Fourier transform is extremely well-suited to study periodicity and density properties of real or complex valued functions. In the same way, its binary counterpart, the Walsh transform plays a fundamental role in the analysis of genetic algorithms (GAs) $[5,7$,

Permission to make digital or hard copies of all or part of this work for personal or classroom use is granted without fee provided that copies are not made or distributed for profit or commercial advantage and that copies bear this notice and the full citation on the first page. To copy otherwise, to republish, to post on servers or to redistribute to lists, requires prior specific permission and/or a fee.

GECCO'05, June 25-29, 2005, Washington, DC, USA.

Copyright 2005 ACM 1-59593-010-8/05/0006 ...\$5.00.
11]. Indeed, they allow for a practical description of epistasis [13]. This notion of epistasis measures, roughly speaking, the presence of links between separate bits in the codified version of the data to be optimized. Although, epistasis alone in no way suffices to predict GA-hardness, it has been shown to correctly correlate to GA-hardness within several particular classes of fitness functions $([9,13])$.

Schemata (hyperplanes in the search space) automatically appear when fitness functions are constructed and the working of the crossover operator is considered. The partition coefficients $\varepsilon(H)$, associated to any schema $\mathrm{H}$, have been used (see $[1,12]$ ) in the analysis and construction of problems that are difficult to solve with a genetic algorithm and also to characterize static deception in a problem. Earlier papers by Goldberg [3, 4] and Heckendorn and Whitley [6] connected schemata and partition coefficients to the Walsh transforms revealing important properties for the understanding of GAs.

On the other hand, although binary encodings are "standard" in the context of GAs, sometimes it is much more natural to use a different type of encoding over not necessarily binary alphabets $([2,10])$. This work extends the results of $[3,4,6]$ to functions over multary alphabets, i.e., functions over the space of strings $\Omega_{n}=\{0, \ldots, n-1\}^{\ell}$, with $n \geq 2$. Actually, our approach also yields more compact and elegant proofs for the aforementioned results.

\section{GENERALIZED WALSH TRANSFORMS- FIRST APPROACH}

We will denote by $\Omega_{2}$ the set $\{0,1\}^{\ell}$ of length $\ell$ binary strings - we fix $\ell$ throughout this text. We will sometimes identify elements $s=s_{\ell-1} \cdots s_{0} \in \Omega_{2}$ with their numerical value, viewing $s$ as the binary expression of some integer $0 \leq s<2^{\ell}$.

To any $t \in \Omega_{2}$ one associates the so-called Walsh function $\psi_{t}$, defined by

$$
\psi_{t}(s)=(-1)^{s \cdot t}=\prod_{i=0}^{\ell-1}(-1)^{s_{i} t_{i}}
$$


where $s \cdot t=\sum_{i=0}^{\ell-1} s_{i} t_{i}$ is the scalar product of $s, t \in \Omega_{2}$. For example, $\psi_{11011}(01100)=-1$ and $\psi_{11011}(01010)=1$. Note that $\psi_{t}(s)$ can be 1 or -1 depending on the number of loci where both $s$ and $t$ have value 1 . Alternatively, define the conjunction $\wedge$ in $\Omega_{2}$ by

$$
s \wedge t=\left(s_{\ell-1} \wedge t_{\ell-1}\right) \ldots\left(s_{0} \wedge t_{0}\right),
$$

where $s_{i} \wedge t_{i}$ is just the product $s_{i} \cdot t_{i}$, for all $s_{i}, t_{i} \in\{0,1\}$ and denote by $u(s)$ the number of loci with value 1 in any string $s$. Then it is easy to see that

$$
\psi_{t}(s)=(-1)^{u(s \wedge t)} .
$$

In order to generalize Walsh coefficients to a multary alphabet $\Sigma=\{0,1, \cdots, n-1\}$, we have at least two possibilities. The first one is inspired by the discrete Fourier transform. We consider the primitive root of unity $r=e^{\frac{2 \pi}{n} i}=$ $\cos \left(\frac{2 \pi}{n}\right)+i \sin \left(\frac{2 \pi}{n}\right)$ (note, that for $n=2$, we have $r=-1$ ) and define, for any $s \in \Omega_{n}=\Sigma^{\ell}$, the Walsh function $\psi_{s}$ on $\Omega_{n}$ by putting

$$
\psi_{s}(t)=r^{s \cdot t},
$$

for every $t \in \Omega_{n}$. Here $s \cdot t$ denotes the scalar product modulo $n$ of $s$ and $t$ in $\Omega_{n}$, i.e.,

$$
s \cdot t=\sum_{i=0}^{\ell-1} s_{i} \cdot t_{i}(\bmod n) .
$$

For example, if $n=3$ and $s=012, t=121$, we have that:

$$
\psi_{s}(t)=r^{(0+2+2)(\bmod 3)}=r
$$

where $r=-\frac{1}{2}+i \frac{\sqrt{3}}{2}$.

Obviously, for any $s, t \in \Omega_{n}$, it follows that

$$
\psi_{s}(t)=\psi_{t}(s)
$$

We may now prove the following multary version of the Balanced Sum Theorem (see [6] for the binary version).

Theorem 1 (First Balanced Sum Theorem). For any $t \in \Omega_{n}$, we have

$$
\sum_{s \in \Omega_{n}} \psi_{t}(s)=\sum_{s=0}^{n^{\ell}-1} \psi_{t}(s)= \begin{cases}n^{\ell} & \text { if } t=0 \\ 0 & \text { otherwise }\end{cases}
$$

Proof. Clearly

$$
\sum_{s \in \Omega_{n}} \psi_{0}(s)=\sum_{s \in \Omega_{n}} 1=n^{\ell}
$$

Now, if $t \neq 0$, suppose that $t_{\ell-1} \neq 0$, and denote, $s=s_{\ell-1} \widehat{s}$ and $t=t_{\ell-1} \widehat{t}$, with $\widehat{s}, \widehat{t} \in \Sigma^{\ell-1}=\widehat{\Omega_{n}}$. Then:

$$
\begin{aligned}
\sum_{s \in \Omega_{n}} \psi_{t}(s)= & \sum_{s \in \Omega_{n}} r^{s_{\ell-1} t_{\ell-1}} r^{\widehat{s} \widehat{t}} \\
= & \sum_{\substack{s \in \Omega_{n} \\
s_{\ell-1}=0}} \psi_{\widehat{t}}(\widehat{s})+r^{t_{\ell-1}} \sum_{\substack{s \in \Omega_{n} \\
s_{\ell-1}=1}} \psi_{\widehat{t}}(\widehat{s})+\cdots \\
& +r^{(n-1) t_{\ell-1}} \sum_{\substack{s \in \Omega_{n} \\
s_{\ell-1}=n-1}} \psi_{\hat{t}}(\widehat{s}) \\
= & \left(1+r^{t_{\ell-1}}+\cdots+r^{(n-1) t_{\ell-1}}\right) \sum_{\widehat{s} \in \widehat{\Omega_{n}}} \psi_{\hat{t}}(\widehat{s}) \\
= & 0 .
\end{aligned}
$$

If we denote by $\widetilde{s}=\widetilde{s}_{\ell-1} \ldots \widetilde{s}_{0}$, the string with $s_{i}+\widetilde{s}_{i}=$ $0(\bmod n)$, for any $s=s_{\ell-1} \cdots s_{0} \in \Omega_{n}$, it follows immediately from the previous result:

Corollary 1.

$$
\sum_{s \in \Omega_{n}} \psi_{t}(s) \psi_{t^{\prime}}(s)= \begin{cases}n^{\ell} & \text { if } t^{\prime}=\widetilde{t} \\ 0 & \text { otherwise. }\end{cases}
$$

Now, if we define, for any $t \in \Omega_{n}$ and any function $f$ : $\Omega_{n} \rightarrow \mathbb{R}$, the corresponding complex Walsh coefficient

$$
v_{t}^{c}=n^{-\ell} \sum_{s \in \Omega_{n}} f(s) \psi_{t}(\widetilde{s})=n^{-\ell} \sum_{s \in \Omega_{n}} f(s) \psi_{\widetilde{s}}(t),
$$

we can prove that the relation between the function $f$ and these coefficients is given by:

$$
f(s)=\sum_{t \in \Omega_{n}} \psi_{t}(s) v_{t}^{c}
$$

for all $s \in \Omega_{n}$. Indeed, if $s \in \Omega_{n}$, then

$$
\begin{aligned}
n^{\ell} \sum_{t \in \Omega_{n}} \psi_{t}(s) v_{t}^{c} & =\sum_{t \in \Omega_{n}} \psi_{t}(s) \sum_{a \in \Omega_{n}} f(a) \psi_{\widetilde{a}}(t) \\
& =\sum_{a \in \Omega_{n}} \sum_{t \in \Omega_{n}}\left(\psi_{s}(t) \psi_{\widetilde{a}}(t)\right) f(a) \\
& =n^{\ell} f(s) .
\end{aligned}
$$

\section{GENERALIZED WALSH TRANFORMS - SECOND APPROACH}

As the sum in the First Balanced Sum Theorem is taken over the whole space $\Omega_{n}$, one may wonder what happens if one restricts $s$ to a schema (or hyperspace) $H$ in $\Omega_{n}$. A schema is usually written as a string over $\{0, \ldots, n-1, \#\}$. This is equivalent to $H$ belonging to $\{0, \ldots, n-1, \#\}^{\ell}$, where \# plays the role of a wild-card symbol. An individual $s_{\ell-1} \ldots s_{0}$ belongs to a schema $H=h_{\ell-1} \ldots h_{0}$ if $s_{i}=h_{i}$ for all $i$ such that $h_{i} \neq \#$. For example, if $n=3$ and $H=1 \# 20$, then the strings of $H$ are:

$$
H=\{1020,1120,1220\}
$$

The order $o(H)$ of $H$ is the number of fixed positions in $H$ (the number of non-wild-card symbols in the schema). If we denote by $|H|$ the cardinality of $H$ (the number of strings "in" or "satisfying" $H)$, then $|H|=n^{\ell-o(H)}$. For the above schema $H=1 \# 20$, we have $o(H)=3$ and, as we have seen $|H|=3^{4-3}=3$.

In order to obtain another generalized Walsh transform, let us start by defining the partition coefficient $\varepsilon(H)$ of a schema $H$ over the multary alphabet $\Sigma$. Exactly as in the binary case $([6])$, we want these $\varepsilon(H)$ to satisfy the general partition equation

$$
f(H)=\sum_{H^{\prime} \supset H} \varepsilon\left(H^{\prime}\right)
$$

where, $H^{\prime} \supset H$ means that $H$ and $H^{\prime}$ agree on the fixed positions of $H^{\prime}$. For example, if $n=3$, then $H=1 \# 2 \subset$ $H^{\prime}=1 \# \#$.

As might be expected, the partition coefficient of the schema $\# \ldots \#$ should be the average of the function $f$. On the other hand, the average value of any schema $H$ of order 1 can be approximated by the schema \#...\#, and the difference between this approximation and its correct value 
will be the partition coefficient of $H$. Let us now consider a schema $H$ of order 2, for example $H=\# \ldots \# a b$, with $a, b \in \Sigma=\{0,1, \ldots, n-1\}$. We may interpret $H$ as a combination of schemata $H^{\prime}$ of order 1 such that $H^{\prime} \supset H$. So, as a first approximation, we could calculate the fitness value of $H$ as follows:

$$
\begin{aligned}
f(H) \approx & f(\# \ldots \#)+(f(\# \ldots \#)-f(\# \ldots a \#)) \\
& +(f(\# \ldots \#)-f(\# \ldots \# b)) .
\end{aligned}
$$

Obviously, in this approximation we ignore the interaction between the fixed positions in $H$. In fact, the difference between the actual value of $f(H)$ and the above approximation is exactly the partition coefficient of $H=\# \ldots \# a b$ :

$$
\begin{aligned}
f(\# \ldots \# a b)= & \varepsilon(\# \ldots \#)+\varepsilon(\# \ldots a \#) \\
& +\varepsilon(\# \ldots \# b)+\varepsilon(\# \ldots \# a b) .
\end{aligned}
$$

Since $\varepsilon(\# \ldots \#)=f(\# \ldots \#)$, iterating the process and rearranging the terms in the general partition equation yields, for any schema $H$, that

$$
\varepsilon(H)=f(H)-\sum_{H^{\prime} \supsetneqq H} \varepsilon\left(H^{\prime}\right),
$$

so the $\varepsilon(H)$ may be calculated recursively. For example:

$$
\varepsilon(\# \ldots a \#)=f(\# \ldots a \#)-\varepsilon(\# \ldots \#) .
$$

In order to recover the original fitness function from the partition coefficients, we will need the following two results of Mason (see [12] for details):

THEOREM 2. For any $H=h_{\ell-1} \ldots h_{i+1} \# h_{i-1} \ldots h_{0}$ (with $\left.h_{j} \in \Sigma \cup\{\#\}\right)$,

$$
\sum_{a \in \Sigma} \varepsilon\left(h_{\ell-1} \ldots h_{i+1} a h_{i-1} \ldots h_{0}\right)=0 .
$$

THEOREM 3. If some $\varepsilon(\cdot)$ satisfies theorem 2, then the general partition equation is satisfied for the fitness values

$$
f(s)=\sum_{s \in H} \varepsilon(H) \text {. }
$$

Theorem 2 shows that the partition coefficients are not independent of each other. We will consider a minimal collection of partition coefficients which generates the set of all partition coefficients and which, by theorem 3, also permits to recover the original fitness function. This minimal set will consist of the so-called generalized natural Walsh coefficients.

We proceed as follows. Denote by $\Sigma^{\prime}$ the alphabet $\Sigma$ augmented with the "don't care" symbol \# and consider the function $\widehat{\beta}$ which associates with any schema $H=h_{\ell-1} \ldots h_{0} \in$ $\left(\Sigma^{\prime} \backslash\{0\}\right)^{\ell}$ the string $\widehat{\beta}(H)=\widehat{\beta}_{\ell-1} \ldots \widehat{\beta}_{0} \in \Sigma^{\ell}$, with

$$
\widehat{\beta}_{i}= \begin{cases}0 & \text { if } h_{i}=\# \\ h_{i} & \text { otherwise }\end{cases}
$$

With each of these $n^{\ell}$ schemata $H$ or, equivalently, the corresponding values $\widehat{\beta}(H)$, we associate a generalized natural Walsh coefficient

$$
v_{H}^{n a t}=v_{\widehat{\beta}(H)}^{n a t}=\varepsilon(H) .
$$

For example, if we consider the schema $H=12 \#$ over a ternary alphabet, then

$$
v_{H}^{n a t}=v_{\widehat{\beta}(12 \#)}^{n a t}=v_{15}^{n a t} .
$$

If $H$ is any schema, we may now define recursively (in the order of $H$ ), the value $v_{H}^{\text {nat }}$ by

$v_{H}^{n a t}= \begin{cases}(-1)^{o(H)} v_{\widehat{\beta}(H)}^{n a t} & \text { if } h_{i} \in \Sigma^{\prime} \backslash\{0\} \\ \sum_{a \in\{1, \ldots, n-1\}}-v_{h_{\ell-1} \ldots h_{i+1} a h_{i-1} \ldots h_{0}}^{\text {nat }} & \text { if } h_{i}=0 .\end{cases}$

Now, if $H$ is any schema, the value $\varepsilon(H)$ is given by

$$
\varepsilon(H)=v_{H}^{n a t} .
$$

The function $v^{\text {nat }}$ satisfies the condition of theorem 2 and, by theorem 3, the general partition equation holds, i.e.,

$$
f(s)=\sum_{s \in H} v_{H}^{n a t} .
$$

So, it thus follows, exactly as in (1) that the original function $f$ may be reconstructed when these general Walsh coefficients $v_{H}^{n a t}$ are known. Now, we can define, for any schema $H \in \Sigma^{\ell}=\Omega_{n}$, a new schema $\beta(H)$ as follows:

$$
\beta(H)_{i}= \begin{cases}0 & \text { if } h_{i}=\#, \\ h_{i} & \text { otherwise. }\end{cases}
$$

It is obvious that $\beta$ and $\widehat{\beta}$ are the same if $H=h_{\ell-1} \ldots h_{0} \in$ $\left(\Sigma^{\prime} \backslash\{0\}\right)^{\ell}$.

Let us give some examples. For $n=3$ and $\ell=1$ the connection between the schemata and the general Walsh coefficients is given by

\begin{tabular}{|c|c|c|}
\hline$H$ & $\beta(H)$ & $v_{H}^{\text {nat }}$ \\
\hline$\#$ & 0 & $v_{0}^{\text {nat }}$ \\
0 & 0 & $v_{1}^{\text {nat }}+v_{2}^{\text {nat }}$ \\
1 & 1 & $-v_{1}^{\text {nat }}$ \\
2 & 2 & $-v_{2}^{\text {nat }}$ \\
\hline
\end{tabular}

and for $n=3$ and $\ell=2$

\begin{tabular}{|c|c|c|}
\hline$H$ & $\beta(H)$ & $v_{H}^{\text {nat }}$ \\
\hline$\# \#$ & 00 & $v_{0}^{\text {nat }}$ \\
$\# 0$ & 00 & $v_{1}^{\text {nat }}+v_{2}^{\text {nat }}$ \\
$\# 1$ & 01 & $-v_{1}^{\text {nat }}$ \\
$\# 2$ & 02 & $-v_{2}^{\text {nat }}$ \\
$0 \#$ & 00 & $v_{3}^{\text {nat }}+v_{6}^{\text {nat }}$ \\
00 & 00 & $v_{4}^{\text {nat }}+v_{5}^{\text {nat }}+v_{7}^{\text {nat }}+v_{8}^{\text {nat }}$ \\
01 & 01 & $-v_{4}^{\text {nat }}-v_{7}^{\text {nat }}$ \\
02 & 02 & $-v_{5}^{\text {nat }}-v_{8}^{\text {nat }}$ \\
$1 \#$ & 10 & $-v_{3}^{\text {nat }}$ \\
10 & 10 & $-v_{4}^{\text {nat }}-v_{5}^{\text {nat }}$ \\
11 & 11 & $v_{4}^{\text {nat }}$ \\
12 & 12 & $v_{5}^{\text {nat }}$ \\
$2 \#$ & 20 & $-v_{6}^{\text {nat }}$ \\
20 & 20 & $-v_{7}^{\text {nat }}-v_{8}^{\text {nat }}$ \\
21 & 21 & $v_{7}^{\text {nat }}$ \\
22 & 22 & $v_{8}^{\text {nat }}$ \\
\hline
\end{tabular}

An explicit relation between both generalized Walsh coefficients $\left(v_{H}^{n a t}\right.$ and $\left.v_{t}^{c}\right)$ can be obtained making use of basic techniques of Linear Algebra (see [8] for details).

\section{SCHEMA AVERAGES AND PARTITION COEFFICIENTS - MULTARY CASE}

Let us first derive the analogues of Theorem 1 and Corollary 1 when we restrict to a schema $H$ in $\Omega_{n}$. We define, for 
any schema $H$ in $\Omega_{n}$,

$$
J(H)=\left\{t \in \Omega_{n} ; h_{i}=\# \Rightarrow t_{i}=0\right\} .
$$

Then, we can prove:

Lemma 1. For any schema $H$ in $\Omega_{n}$, for any $s \in H$ and any $t \in J(H)$, we have

$$
\psi_{t}(s)=\psi_{t}(\beta(H)) .
$$

ProOF.

$$
\psi_{t}(s)=\prod_{i=0}^{\ell-1} r^{s_{i} t_{i}}=\prod_{i ; h_{i}=\#} r^{s_{i} t_{i}} \prod_{i ; h_{i} \neq \#} r^{s_{i} t_{i}} .
$$

As $t \in J(H)$, we have $t_{i}=0$ if $h_{i}=\#$ and $\prod_{i ; h_{i}=\#} r^{s_{i} t_{i}}=1$. On the other hand, as $s \in H$ we have $s_{i}=h_{i}=\beta(H)_{i}$, for all $i$ with $h_{i} \neq \#$. We thus obtain

$$
\psi_{t}(s)=\prod_{i ; h_{i} \neq \#} r^{s_{i} t_{i}}=\psi_{t}(\beta(H)),
$$

as claimed.

Theorem 4 (Second Balanced Sum Theorem). For any $t \in \Omega_{n}$ and any schema $H$ over $\Omega_{n}$, we have

$$
\sum_{s \in H} \psi_{t}(s)= \begin{cases}|H| \psi_{t}(\beta(H)) & \text { if } t \in J(H) \\ 0 & \text { if } t \notin J(H) .\end{cases}
$$

Proof. If $t \in J(H)$, the assertion follows from lemma 1. If $t \notin J(H)$, then there exists at least, one non-zero component of $t$, say $t_{i}$, with $h_{i}=\#$. Let us suppose that $i=\ell-1$, then, arguing as in Theorem 1, it follows that $\sum_{s \in H} \psi_{t}(s)=0$, indeed.

Also, we can obtain:

Corollary 2. For any schema $H$ over $\Omega_{n}$, we have

$$
f(H)=\sum_{t \in J(H)} v_{t}^{c} \psi_{t}(\beta(H)) .
$$

In particular,

$$
f\left(\Omega_{n}\right)=f(\# \ldots \#)=n^{-\ell / 2} v_{0}^{c} .
$$

EXAMPLE 1. Let us include some examples of the relation between $f$ and its general Walsh coefficients.

Let $H_{a}$ be the schema $H_{a}=\# \ldots \# a \# \ldots \#$ with $a \in \Sigma$ at position $i$. Then $\beta\left(H_{a}\right)=0 \ldots 0 a 0 \ldots 0$ and $J\left(H_{a}\right)=$ $0 \cdots 0 \# 0 \cdots 0=\left\{k n^{i} ; 0 \leq k<n\right\}$. So, for $t=k n^{i} \in J\left(H_{a}\right)$,

$$
\psi_{t}\left(\beta\left(H_{a}\right)\right)=r^{k \cdot a}
$$

and

$$
\begin{aligned}
f\left(H_{a}\right) & =n^{-\ell / 2} \sum_{t \in J\left(H_{a}\right)} w_{t} \psi_{t}\left(\beta\left(H_{a}\right)\right) \\
& =n^{-\ell / 2}\left(w_{0}+r^{a} \cdot w_{n^{i}}+\cdots+r^{(n-1) a} \cdot w_{(n-1) n^{i}}\right),
\end{aligned}
$$

i.e.,

$$
f\left(H_{a}\right)=n^{-\ell / 2} \sum_{k=0}^{(n-1)} r^{k \cdot a} \cdot w_{k \cdot n^{i}} .
$$

Now, consider the order 2 schema

$$
H_{a b}=\# \ldots \stackrel{i}{i}_{a} \# \ldots \stackrel{j}{b}^{\downarrow} \# \cdots \#
$$

with $a, b \in \Sigma$.

In this case,

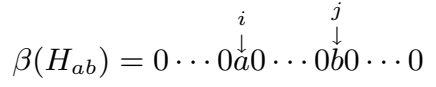

and

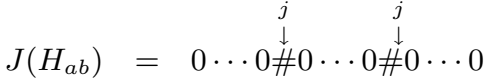

$$
\begin{aligned}
& =\left\{p n^{i}+q n^{j} ; 0 \leq p, q<n\right\}
\end{aligned}
$$

So, for all $t \in J\left(H_{a b}\right)$

$$
\psi_{t}\left(\beta\left(H_{a b}\right)\right)=r^{a t_{i}+b t_{j}}=r^{a p+b q},
$$

for some $0 \leq p, q<n$. We thus obtain:

$$
\begin{aligned}
f\left(H_{a b}\right) & =n^{-\ell / 2} \sum_{t \in J\left(H_{a b}\right)} w_{t} \psi_{t}\left(\beta\left(H_{a b}\right)\right) \\
& =n^{-\ell / 2} \sum_{p, q=0}^{(n-1)} r^{a p+b q} \cdot w_{p \cdot n^{i}+q \cdot n^{j}}
\end{aligned}
$$

Finally, we want to establish a link between partition coefficients and the general Walsh coefficients $\left(v_{t}^{c}\right)$ defined in the first section. As there is no possible ambiguity, we will use the notation $v_{t}$ when we refer to these Walsh coefficients.

We start by defining

$$
\alpha(H)=\left\{t \in \Omega_{n} ; h_{i}=\# \Leftrightarrow t_{i}=0\right\} .
$$

We will need the following technical lemmas.

Lemma 2. For any pair of schemata $H, H^{\prime} \in \Sigma^{\ell}$, we have

1. if $H^{\prime} \supset H$ then $\alpha\left(H^{\prime}\right) \subset J(H)$;

2. for any $t \in J(H)$, there exists exactly one $H^{\prime} \supset H$ with $t \in \alpha\left(H^{\prime}\right)$.

Proof. ¿From the definition of $\alpha$ it follows that

$$
t \in \alpha\left(H^{\prime}\right) \Leftrightarrow\left(h_{i}^{\prime}=\# \Leftrightarrow t_{i}=0 \text {, for all } 0 \leq i<\ell\right) .
$$

So, as $H^{\prime} \supset H$, if $h_{i}=\#$ then $h_{i}^{\prime}=\#$ and we have that $t_{i}=0$. This implies that $t \in J(H)$.

To prove the existence of an $H^{\prime}$ for the second statement, we define $H^{\prime}=h_{\ell-1}^{\prime} \cdots h_{0}^{\prime}$ by

$$
h_{i}^{\prime}= \begin{cases}\# & t_{i}=0 \\ h_{i} & t_{i} \neq 0\end{cases}
$$

Obviously, $H^{\prime} \supset H$. On the other hand, as $t \in J(H)$, then $t \in \alpha\left(H^{\prime}\right)$. Indeed, if $t_{i} \neq 0$ then we have that $h_{i}^{\prime} \neq \#$ and so $h_{i}^{\prime}=h_{i} \neq \#$. The fact that $h_{i}^{\prime}=\#$, when $t_{i}=0$ follows directly from the definition of $H^{\prime}$.

Moreover, $H^{\prime}$ is unique as such. In fact, if $t_{i}=0$ then $h_{i}^{\prime}=\#$, as $t \in \alpha\left(H^{\prime}\right)$.

If $t_{i} \neq 0$ then, $h_{i} \neq \#$ (as $\left.t \in J(H)\right)$ and necessarily $h_{i}^{\prime} \neq \#$ (because $h_{i}^{\prime}=\#$ would imply $t_{i}=0$ ). So, as $H^{\prime} \supset H$, it follows that $h_{i}^{\prime}=h_{i}$.

Lemma 3. For any schema $H$ over a multary alphabet, we have

$$
\left.J(H)=\bigcup_{H^{\prime} \supset H} \alpha\left(H^{\prime}\right) \quad \text { (disjoint union }\right) .
$$


ProOF. Note that it only remains to prove that for any pair of schemata $H^{\prime}, H^{\prime \prime} \supset H$ we have $H^{\prime} \neq H^{\prime \prime} \Leftrightarrow \alpha\left(H^{\prime}\right) \cap$ $\alpha\left(H^{\prime \prime}\right)=\emptyset$. If $\alpha\left(H^{\prime}\right) \cap \alpha\left(H^{\prime \prime}\right) \neq \emptyset$ then, by picking $t \in$ $\alpha\left(H^{\prime}\right) \cap \alpha\left(H^{\prime \prime}\right)$, we have that $t \in J(H)$ and the unicity given in lemma 2 implies $H^{\prime}=H^{\prime \prime}$.

The fact that $H^{\prime}=H^{\prime \prime}$ implies $\alpha\left(H^{\prime}\right) \cap \alpha\left(H^{\prime \prime}\right) \neq \emptyset$ is obvious.

Lemma 4. For any $H^{\prime} \supset H$ and for any $t \in \alpha\left(H^{\prime}\right)$ we have $\psi_{t}(\beta(H))=\psi_{t}\left(\beta\left(H^{\prime}\right)\right)$.

Proof. Let us check that $t \cdot \beta(H)=t \cdot \beta\left(H^{\prime}\right)$. It suffices to verify for all $i$ that $t_{i} \cdot \beta_{i}=t_{i} \cdot \beta_{i}^{\prime}$, when we write $\beta_{i}$ (resp. $\left.\beta_{i}^{\prime}\right)$ for $\beta(H)_{i}\left(\operatorname{resp} . \beta\left(H^{\prime}\right)_{i}\right)$.

If $h_{i}=\#$ then $\beta_{i}=0$ and also $h_{i}^{\prime}=\#$, so $\beta_{i}^{\prime}=0$.

If $h_{i} \neq \#$, as $H^{\prime} \supset H$ we need to distinguish two cases:

1. if $h_{i}^{\prime}=h_{i}$ then $\beta_{i}=h_{i}=h_{i}^{\prime}=\beta_{i}^{\prime}$;

2. if $h_{i}^{\prime}=\#$ then $t_{i}=0\left(\right.$ as $\left.t \in \alpha\left(H^{\prime}\right)\right)$.

This proves the assertion.

Finally, we reach our aim with the following theorem which states that the partition coefficients of any schema $H$ may be computed using only these Walsh coefficients $v_{t}$ for which the number of loci with value different from zero in $t$ is at most equal to the order of $H$.

TheOREM 5. For any schema $H$ over $\Omega_{n}$, we have

$$
\varepsilon(H)=\sum_{t \in \alpha(H)} v_{t} \psi_{t}(\beta(H)) .
$$

Proof. First note that

$$
\begin{aligned}
f(H) & =\sum_{t \in J(H)} v_{t} \psi_{t}(\beta(H)) \\
& =\sum_{H^{\prime} \supset H} \sum_{t \in \alpha\left(H^{\prime}\right)} v_{t} \psi_{t}(\beta(H)) \\
& =\sum_{H^{\prime} \supset H} \sum_{t \in \alpha\left(H^{\prime}\right)} v_{t} \psi_{t}\left(\beta\left(H^{\prime}\right)\right) .
\end{aligned}
$$

Since the result is true for $H=\# \ldots \#($ as $\alpha(H)=$ $\alpha\left(\Omega_{n}\right)=\{0 \cdots 0\}=\beta\left(\Omega_{n}\right), \psi_{0}(\beta(H))=1$ and so $f\left(\Omega_{n}\right)=$ $\left.v_{0}\right)$, we may argue by induction in the order of $H$.

Let us assume that

$$
\varepsilon\left(H^{\prime}\right)=\sum_{t \in \alpha\left(H^{\prime}\right)} v_{t} \psi_{t}\left(\beta\left(H^{\prime}\right)\right)
$$

for all $H^{\prime} \supsetneqq H$. We then have

$$
\begin{aligned}
\varepsilon(H) & =f(H)-\sum_{H^{\prime} \supsetneqq H} \varepsilon\left(H^{\prime}\right) \\
& =\sum_{H^{\prime} \supset H} \sum_{t \in \alpha\left(H^{\prime}\right)} v_{t} \psi_{t}\left(\beta\left(H^{\prime}\right)\right)-\sum_{H^{\prime} \supsetneqq H} \sum_{t \in \alpha\left(H^{\prime}\right)} v_{t} \psi_{t}\left(\beta\left(H^{\prime}\right)\right) \\
& =\sum_{t \in \alpha(H)} v_{t} \psi_{t}(\beta(H)) .
\end{aligned}
$$

EXAmple 2. To conclude, let us include some easy examples of the previous relation. If we denote, as before, by $H_{a}$ the order 1 schema $H_{a}=\# \cdots \stackrel{i}{\perp}_{a}^{\perp} \# \cdots \#$, with $0 \leq a<n$, we already know that

$$
\beta\left(H_{a}\right)=0 \cdots 0 \stackrel{i}{a} 0 \cdots 0
$$

and also that $\psi_{t}\left(\beta\left(H_{a}\right)\right)=r^{a \cdot t_{i}}$. As it can easily be seen that

$$
\begin{aligned}
\alpha\left(H_{a}\right) & =\left\{t=0 \cdots 0 t_{i} 0 \cdots 0 ; t_{i} \neq 0\right\} \\
& =\left\{k n^{i} ; 0<k<n\right\},
\end{aligned}
$$

we obtain:

$$
\varepsilon\left(H_{a}\right)=\sum_{t \in \alpha\left(H_{a}\right)} r^{a \cdot t_{i}} v_{t}=\sum_{k=1}^{n-1} r^{a \cdot k} v_{k \cdot n^{i}}
$$

For example, for ternary alphabets $(n=3)$, we have $r=$ $-\frac{1}{2}+i \frac{\sqrt{3}}{2}$ and

$$
\begin{aligned}
& \varepsilon\left(\# \cdots \# \stackrel{i}{i}{ }^{i} \# \cdots \#\right)=v_{3^{i}}+v_{2 \cdot 3^{i}}, \\
& \varepsilon\left(\# \cdots \# \stackrel{i}{\downarrow}{ }^{\downarrow} \# \cdots \#\right)=r v_{3^{i}}+r^{2} v_{2 \cdot 3^{i}} \text {, } \\
& \varepsilon(\# \cdots \# \stackrel{i}{\stackrel{i}{2}} \# \cdot \cdots \#)=r^{2} v_{3^{i}}+r v_{2 \cdot 3^{i}} .
\end{aligned}
$$

Finally, let us consider again the order 2 schemata

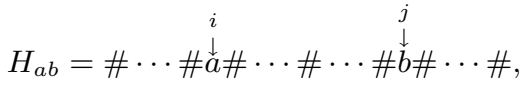

with $a, b \in\{0,1, \ldots, n-1\}$ Then, we already know that

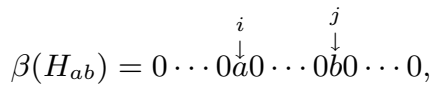

and $\psi_{t}\left(\beta\left(H_{a b}\right)\right)=r^{a \cdot p+b \cdot q}$ for $t \in \Omega_{n}$. Taking into account that

$$
\begin{aligned}
\alpha\left(H_{a b}\right) & =\left\{t=0 \cdots 0 t_{i} 0 \cdots 0 \cdots 0 t_{j} 0 \cdots 0 ; t_{i}, t_{j} \neq 0\right\} \\
& =\left\{p n^{i}+q n^{j} ; 0<p, q<n\right\}
\end{aligned}
$$

we have

$$
\begin{aligned}
\varepsilon\left(H_{a b}\right) & =n^{-\ell / 2} \sum_{t \in \alpha\left(H_{a b}\right)} r^{a \cdot t_{i}+b \cdot t_{j}} w_{t} \\
& =n^{-\ell / 2} \sum_{p, q=1}^{n-1} r^{a \cdot p+b \cdot q} w_{p n^{i}+q n^{j}}
\end{aligned}
$$


For example, if $n=3$ we obtain

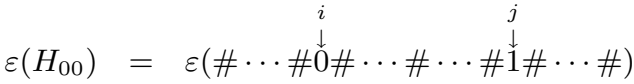

$$
\begin{aligned}
& =3^{-\ell / 2} \sum_{p, q=1}^{2} w_{p \cdot 3^{i}+q \cdot 3^{j}}
\end{aligned}
$$

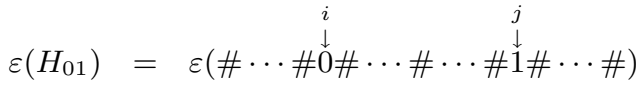

$$
\begin{aligned}
& =3^{-\ell / 2} \sum_{p, q=1}^{2} r^{q} w_{p \cdot 3^{i}+q \cdot 3^{j}}
\end{aligned}
$$

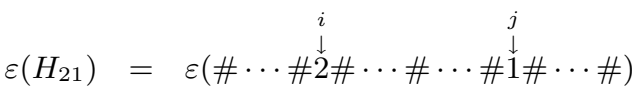

$$
\begin{aligned}
& =3^{-\ell / 2} \sum_{p, q=1}^{2} r^{2 \cdot p+q} w_{p \cdot 3^{i}+q \cdot 3^{j}},
\end{aligned}
$$

for instance.

\section{CONCLUDING REMARKS}

The main purpose of this note was to generalize the machinery of Walsh transforms from binary to multary alphabets, with emphasis on their utility in the calculation of partition coefficients. We included some concrete examples showing the strength, in the multary case as well, of the use of Walsh coefficients in this context. As a nice side-effect, it appeared that our techniques not only provide for a nice extension of "classical" results to the multary case, but also yields shorter and more transparent proofs of the latter.

\section{ACKNOWLEDGMENTS}

This work has been supported by a research grant of Xunta de Galicia (Spain) PGIDT03PXIA10502PR and by GOA "Generic Optimization" at the University of Antwerp.

\section{REFERENCES}

[1] A. D. Bethke, Genetic algorithms as function optimizers (Doctoral dissertation, University of Michigan, 1980) and Dissertation Abstracts International, 41 (9), University Microfilms No. 8106101.

[2] P. Field, A Multary Theory for Genetic Algorithms: Unifying Binary and Nonbinary Problem Representations, Ph.D. Thesis, University of London, London, UK, 1996.
[3] D. E. Goldberg, Genetic Algorithms and Walsh Functions: Part I, A Gentle Introduction, Complex Systems 3 129-152, 1989.

[4] D. E. Goldberg. Genetic Algorithms and Walsh Functions: Part II: Deception and its Analysis. Complex Systems 3:153-171, 1989.

[5] D. E. Goldberg, The Design of Innovation, Kluwer Academic Publishers, Boston, Dordrecht, London, 2002.

[6] R. B. Heckendorn and D. Whitley. Predicting epistasis from mathematical models. Evolutionary Computation, 7(1):69-101, 1999.

[7] J. H. Holland Adaptation in natural and artificial systems, University of Michigan Press, Ann Arbor, 1975 .

[8] M. T. Iglesias, C. Vidal, D. Suys and A. Verschoren, Generalized Walsh Transforms and Epistasis, to appear in Bulletin of Belgian Mathematical Society-Simon Stevin (accepted).

[9] M. T. Iglesias, C. Vidal, D. Suys and A. Verschoren, Epistasis and Unitation, Computers and Artificial Intelligence, 18, 467-483, 1999.

[10] M. T. Iglesias, C. Vidal, D. Suys and A. Verschoren, Multary Epistasis, Bulletin of the Belgian Mathematical Society, Simon Stevin, 8, 651-671, 2001.

[11] L. Kallel, B. Naudts and A. Rogers, Theoretical Aspects of Evolutionary Computing, 2001, Natural Computing Series. Springer-Verlag, Berlin, Heidelberg, New York, 2001.

[12] A. J. Mason, Partition Coefficients, Static Deception and Deceptive Problems for Non-binary Alphabets, in: Proceedings of the 4th International Conference on Genetic Algorithms, eds. R. K. Belew and L. B. Booker, 210-214, Morgan Kaufmann Publishers, 210-214, 1991.

[13] B. Naudts, D. Suys and A. Verschoren, Epistasis, deceptivity and Walsh transforms, in: Proceedings of the International ICSC Symposium on Engineering of Intelligent Systems (EIS'98) Volume 1: Fuzzy Logic / Genetic Algorithms, 210-216, ICSC Academic Press, 1998.

[14] G. J. E. Rawlins, Foundations of Genetic Algorithms, Morgan Kaufmann Publishers, San Mateo, 1991. 